\title{
A Complete Proof System for Timed Observations
}

\author{
Yolanda Ortega-Mallén \\ David de Frutos-Escrig \\ Sección Departamental de Informática y Automática \\ Facultad de Matemáticas \\ Universidad Complutense, 28040 Madrid, Spain*
}

\begin{abstract}
Timed Observations is a failure and divergence semantic model for concurrent processes, suitable for real-time systems. Actions are not instantaneous but need some time to complete their execution, and true concurrency is expressed by action multisets (bags). Time is global and discrete.

The model is applied to TCSP, obtaining a denotational semantics, for which a complete proof system is developed.
\end{abstract}

\section{Introduction}

In [OdF90] a timed failure semantic model for concurrent processes was presented. In that model actions performed by a process are not instantaneous, but need a certain amount of time to complete.

Following the ideas of Milner's [Mil80], that a concurrent process is not an isolated item, but part of a whole system of interacting machines and users, what is interesting about a process is its external behaviour, i.e. what an external observer (an user or another process) can notice. In our model (Timed Observations), the observer not only notices the visible actions performed by the process (traces), but he also relates each action to the instant when it is executed (timed traces). Moreover, he is an active observer and tries to guess the future behaviour of the process by asking it to perform some actions from a set (refusals as in [BR85]). We are also interested in differentiating divergence (the possibility of engaging in an unbounded sequence of internal actions) from deadlock (doing nothing). Thus we make the idealised assumption that divergence is observable (of course, divergence is not computable in general). Therefore this model has a timed failures divergences semantics.

The model induces a specification-oriented denotational semantics in the sense of [OH86] which is applied to TCSP [HBR81].

*Part of the work was developed during a two-month sojourn of the first author as collaborator in the RWTH-Aachen (Lehrstuhl für Informatik II) (FRG) and a short visit of the second author to this same center. 
We have notice of several research groups working on timed models. Some of them have produced denotational or compositional semantics like [KSdR*85,GB87,RR87]; some others have developed timed process algebras or calculi, like [NRSV90,QAF89,MT89, HR90,BB90,Yi90] among others. In the present paper we try to combine both approaches and give an equational proof system for the denotational semantics obtained for TCSP processes. This proof system is proven to be correct and complete.

The paper is structured as follows: section 2 introduces the basic notions and definitions in the model. In section 3 we describe the corresponding denotational semantics for TCSP-processes. In section 4 we present the proof system.

\section{Timed Observations}

The model given here is slightly different from the one presented in [OdF90]. The introduced changes are mainly technical, and arose when developing the proof system. Next we give its main concepts and definitions. The interested reader is referred to [OdF90] and mainly to [Ort90] for extended explanations, and complete proofs of the results given in this section.

\subsection{Action duration}

Let us start defining the kind of things we can observe in a process. For we fix the set of actions which the processes may perform: the finite alphabet $\mathcal{A}$. These actions are considered to be indivisible but not instantaneous, i.e. we assume a duration function $d: \mathcal{A} \longrightarrow \mathbb{N}^{+}$(where $\mathbb{N}^{+}$represents the non-zero integers) which associates to each action the time needed to complete its execution (in temporary units). As actions are no longer instantaneous, hidden actions do not disappear completely from the trace, because its duration time will remain reflected.

\subsection{Action Bags}

As we are interested in a general model for concurrent processes, we need some way to reflect the possibility of having several actions performing simultaneously. The most natural way to express this ${ }^{1}$, is to consider action bags (or multisets). One important restriction is that bags are always finite, corresponding to the fact that the execution of an infinite amount of simultaneous actions is unrealistic. Sets (and multisets) appear in several models for concurrent processes like [Mil83a,Mil83b,TV89,Azc89].

Def. 2.1 The set of bags over an alphabet $\mathcal{A}$ is $\mathcal{B}(\mathcal{A})=\{B: \mathcal{A} \longrightarrow \mathbb{N}\}$.

Let $a \in \mathcal{A}$, some interesting bags and bag sets are defined:

empty bag: $B_{\emptyset} \stackrel{\text { def }}{=} \forall a \in \mathcal{A}, B_{\mathfrak{\vartheta}}(a)=0$,

\footnotetext{
${ }^{1}$ Classical theories for concurrency are based on interleaving semantics where concurrency is reduced to sequentiality + non-determinism. This is derived from the fact that on each process-step only one action is executed. However, more recent works on this topic tend to consider concurrency as a language primitive, giving way to what is known as true concurrency [BC88].
} 
bag generated by $a: B_{a} \stackrel{\text { def }}{=} \forall b \in \mathcal{A}, B_{a}(b)=\left\{\begin{array}{lll}0 & \text { if } & b \neq a \\ 1 & \text { if } & b=a\end{array}\right.$

non-empty bags: $\mathcal{B}^{+}(\mathcal{A})=\mathcal{B}(\mathcal{A})-\left\{B_{\emptyset}\right\}$,

bags containing $a$ : $\mathcal{B}_{a}(\mathcal{A})=\{B \in \mathcal{B}(\mathcal{A}) \mid B(a)>0\}$,

bags different from $B_{a}: \mathcal{B}_{-a}(\mathcal{A})=\mathcal{B}(\mathcal{A})-\left\{B_{a}\right\}$.

When the alphabet is understood, it may be omitted obtaining $\mathcal{B}, \mathcal{B}^{+}, \mathcal{B}_{a}, \ldots$

\subsubsection{Operations on bags}

When defining the semantics we will need some operations and definitions on action bags.

Let $a \in \mathcal{A} ; A \subseteq \mathcal{A} ; B, B_{1}, B_{2} \in \mathcal{B}(\mathcal{A}) ; n \in \mathbb{N}$.

Alphabet: $\mathcal{A}(B)=\{a \in \mathcal{A} \mid B(a)>0\}$.

Size: $|B|=\sum_{a \in \mathcal{A}} B(a)$.

Partial order: $B_{1} \leq_{\mathrm{B}} B_{2} \stackrel{\text { def }}{=} \forall a \in \mathcal{A}, B_{1}(a) \leq B_{2}(a)$.

Addition: $B_{1}+{ }_{\mathrm{B}} B_{2} \stackrel{\text { def }}{=} \forall a \in \mathcal{A}, B_{1}+{ }_{\mathrm{B}} B_{2}(a) \stackrel{\text { def }}{=} B_{1}(a)+B_{2}(a)$,

As an abbreviation we define, $0 * B=B_{\emptyset}$,

$$
(n+1) * B=n * B+_{\mathrm{B}} B .
$$

Hiding: $B \backslash a \stackrel{\text { def }}{=} \forall b \in \mathcal{A}, B \backslash a(b)= \begin{cases}B(b) & \text { if } b \neq a \\ 0 & \text { if } b=a\end{cases}$

Restriction: $B\left\lceil A \stackrel{\text { def }}{=} \forall b \in \mathcal{A}, B\left\lceil A(b)=\left\{\begin{array}{lll}B(b) & \text { if } & b \in A \\ 0 & \text { if } & b \notin A\end{array}\right.\right.\right.$

Synchronization: it is only defined if $B_{1}\left\lceil A=B_{2}\lceil A\right.$,

$$
B_{1} \oplus_{A} B_{2} \stackrel{\text { def }}{=} \forall a \in \mathcal{A}, B_{1} \oplus_{A} B_{2}(a)=\left\{\begin{array}{lll}
B_{1}(a) & \text { if } & a \in A \\
B_{1}(a)+B_{2}(a) & \text { if } & a \notin A
\end{array}\right.
$$

Both bags must coincide on the actions of the synchronization set $A$, but a pair of synchronized actions is reduced to a unique action.

We will use the action bags to generalize the notion of failure [BR85], which embodies traces and refusals. 


\subsection{Timed traces}

Classical traces reflect some causal order on the executed actions. Nevertheless this order is insufficient for the study of real-time system behaviour. As a more absolute time notion is needed, we relate each occurence of an action to the instant when it is produced (more exactly to the instant when it starts, as actions are not instantaneous), obtaining timed traces. Actions can be performed from instant 1 on (instant 0 represents the initial state of the process).

Def. 2.2 The set of timed traces is $\mathcal{T} \mathcal{T}=\left\{t: \mathbb{N}^{+} \longrightarrow \mathcal{B}\right\}$.

An special and useful trace is the empty trace $t_{\emptyset} \stackrel{\text { def }}{=} \forall i \in \mathbb{N}^{+}, t_{\emptyset}(i)=B_{\emptyset}$.

The empty bag now plays an important role, as it represents the passing of time with the process doing no externally visible action. ${ }^{2}$

Def. 2.3 For each $t \in \mathcal{T} \mathcal{T}$, we define

- its first non-empty time instant by $\inf (t f): \inf \left(t_{\varpi}\right)=0$, $\inf (t)=\min \left\{n \mid t(n) \neq B_{\emptyset}\right\}, t \neq t_{\emptyset}$.

- its last non-empty time instant by $\sup (t f): \sup \left(t_{\boxplus}\right)=0$, $\sup (t)=\max \left\{n \mid t(n) \neq B_{\emptyset}\right\}, t \neq t_{\emptyset}$.

From a practical point of view, processes can only be observed during finite time intervals. As a consequence we have the following definition:

Def. 2.4 The set of finite timed traces is $\mathcal{T} \mathcal{T} \mathcal{F}=\{(t, n) \mid t \in \mathcal{T} \mathcal{T} \wedge n \geq \sup (t)\}$.

For each $t f=(t, n) \in \mathcal{T} \mathcal{T} \mathcal{F}$ we define: trace $(t f)=t$ and end $(t f)=n$.

Notice that $\operatorname{end}(t f)=0$ implies trace $(t f)=t_{t}$. The trace $\left(t_{\theta}, 0\right)$ corresponds to the initial state of the process, when the observer has not yet started to observe.

\subsubsection{Operations on traces}

Let $A \subseteq \mathcal{A} ; n \in \mathbb{N}^{+} ; B \in \mathcal{B} ; t \in \mathcal{T} \mathcal{T} ; t f, t_{1}, t_{2} \in \mathcal{T} \mathcal{T} \mathcal{F}$, we define:

Alphabet: $\mathcal{A}(t f)=\bigcup_{1 \leq i \leq e n d(t f)} \mathcal{A}(\operatorname{trace}(t f)(i))$.

Adding traces: $t_{1}+\mathrm{T} t_{2} \stackrel{\text { def }}{=}\left\{\begin{array}{l}\forall i \in \mathbb{N}^{+}, \text {trace }\left(t_{1}+\mathrm{T} t_{2}\right)(i)=\operatorname{trace}\left(t_{1}\right)(i)+{ }_{\mathrm{B}} \operatorname{trace}\left(t_{2}\right)(i) \\ \operatorname{end}\left(t_{1}+\mathrm{T} t_{2}\right)=\max \left\{\operatorname{end}\left(t_{1}\right), \text { end }\left(t_{2}\right)\right\}\end{array}\right.$

\footnotetext{
${ }^{2}$ Unlike in [TV89] where empty bags are only included to facilitate the definition of the synchronizing operator, but they can be arbitrarily added or eliminated.
} 


\section{Adding a bag to a trace:}

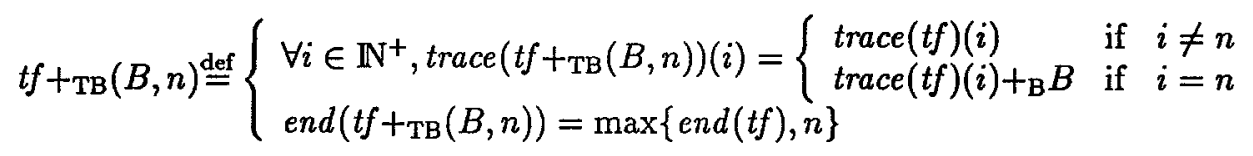

We define the trace including only one action: $t_{n, \alpha} \stackrel{\text { def }}{=} \operatorname{trace}\left(\left(t_{\otimes}, 0\right)+\mathrm{TB}\left(B_{a}, n\right)\right)$.

\section{Moving along time:}

$$
\operatorname{mov}(t f, n) \stackrel{\text { def }}{=} \begin{cases}\forall i \in \mathbb{N}^{+}, \operatorname{trace}(\operatorname{mov}(t f, n))(i)= \begin{cases}\operatorname{trace}(t f)(i-n) & \text { if } i>n \\ B_{\emptyset} & \text { if } i \leq n \\ \operatorname{end}(\operatorname{mov}(t f, n))=\operatorname{end}(t f)+n\end{cases} & \end{cases}
$$

Concatenation: $t_{1} \cdot t_{2} \stackrel{\text { def }}{=} t_{1}+{ }_{T} \operatorname{mov}\left(t_{2}, \operatorname{end}\left(t_{1}\right)\right)$.

Stretch: Stretch $(t f)^{3}$,

$$
\begin{aligned}
& \operatorname{Stretch}\left(t_{\emptyset}, n\right)=\left\{\left(t_{\emptyset}, m\right) \mid m \geq n\right\}, \\
& \operatorname{Stretch}((t, n)+\mathrm{TB}(B, n+1))= \\
& \left\{t f+_{\mathrm{TB}} \sum_{k=1}^{m}\left(B_{k}, n_{k}\right) \mid \sum_{k=1}^{m} B_{k}=B \wedge n_{k} \geq \operatorname{end}(t f)+1 \wedge t f \in \operatorname{Stretch}(t, n)\right\} .
\end{aligned}
$$

Actions in a bag may be "stretched" and executed at different time instants. The only restriction for it is to keep the relative order between bags in the original trace.

Hiding: $t f \backslash a \stackrel{\text { def }}{=}\left\{\begin{array}{l}\forall i \in \mathbb{N}^{+}, \operatorname{trace}(t f \backslash a)(i)=\operatorname{trace}(t f)(i) \backslash a \\ e n d(t f \backslash a)=e n d(t f)\end{array}\right.$

Restriction to an action set: $\operatorname{tf}\left\lceil A \stackrel{\text { def }}{=}\left\{\begin{array}{l}\forall i \in \mathbb{N}^{+}, \operatorname{trace}(t f\lceil A)(i)=\operatorname{trace}(t f)(i)\lceil A \\ \operatorname{end}(t f\lceil A)=\operatorname{end}(t f)\end{array}\right.\right.$

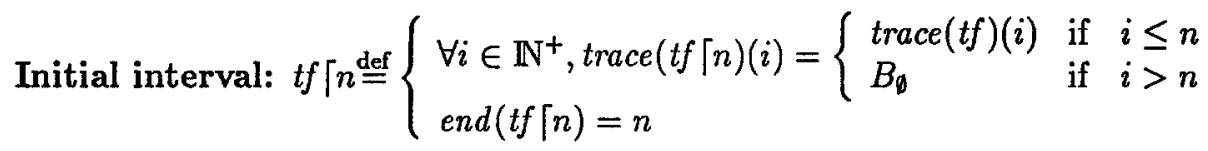

Synchronization: it is only defined for traces verifying $t_{1}\left\lceil A=t_{2}\lceil A\right.$.

$$
t_{1} \oplus_{A} t_{2} \stackrel{\text { def }}{=}\left\{\begin{array}{l}
\forall i \in \mathbb{N}^{+}, \operatorname{trace}\left(t_{1} \oplus_{A} t_{2}\right)(i)=\operatorname{trace}\left(t_{1}\right)(i) \oplus_{A} \operatorname{trace}\left(t_{2}\right)(i) \\
\text { end }\left(t_{1} \oplus_{A} t_{2}\right)=\operatorname{end}\left(t_{1}\right)\left(=\operatorname{end}\left(t_{2}\right)\right)
\end{array}\right.
$$

Operators on finite timed traces can also be applied to non-finite timed traces.

\footnotetext{
${ }^{3}$ This operation is borrowed from [TV89].
} 


\subsection{Timed failures}

Failures consist on traces plus a refusal set. A refusal is a finite set of non-empty action bags. These sets can be empty, but empty bags are not taken into account, because refusing the empty bag means that the process refuses to do nothing, but our model supports "no maximum parallelism" [SM81], i.e. actions can arbitrarily delay its execution, so that every process can always choose to let the time pass doing nothing! We should point out that this model is a general framework for timed processe, and that it can be convenientely modified to meet other desired timing requirements, like it was done in [OdF90], where two versions were presented: one introducing time-outs and the other requiring internal actions to be executed as soon as possible.

Def. 2.5 The set of refusals is $\mathcal{R}=\left\{r \in \mathcal{P} \mathcal{F}\left(\mathcal{B}^{+}\right)\right\}$.

Def. 2.6 Let $r_{1}, r_{2} \in \mathcal{R}$ we define its synchronization:

$r_{1} \oplus_{A} r_{2} \stackrel{\text { def }}{=} \mathcal{P} \mathcal{F}\left(\left\{B \in \mathcal{B}^{+} \mid \forall B_{1}, B_{2}: B=B_{1} \oplus_{A} B_{2} \Rightarrow B_{1} \in r_{1} \vee B_{2} \in r_{2}\right\}\right)$.

Def. 2.7 The set of timed failures is $\mathcal{F} \mathcal{T}=\{\langle t f, r\rangle \mid t f \in \mathcal{T} \mathcal{T} \mathcal{F} \wedge r \in \mathcal{R}\}$.

For each $f=\langle t f, r\rangle \in \mathcal{F} \mathcal{T}$ we define: $T(f)=t f$ and $R(f)=r$.

\subsection{Divergence}

A process is said to diverge when it is engaged in an unbounded sequence of internal actions. The divergence in our model is not catastrophic; i.e. the feasibility of divergence is not necessarily permanent, it may disappear as the process evolves by performing further actions, i.e. by choosing a non-diverging path. Therefore we do not identify a possibly diverging process with chaos (the process which has every possible behaviour). ${ }^{4}$

Obviously it is very convenient to be able to distinguish a never diverging process from one which has this possibility. Therefore, we suppose that our notion of observer is very powerful, and the possibility of divergence in a process can be externally detected.

\subsection{Timed observations}

Putting all the above concepts together, the observable behaviour of a process consists of a timed failure set plus a divergence set (timed traces which can be extended with an infinite sequence of internal actions).

Def. 2.8 The set of timed observations $\mathcal{O}$ is:

$$
\mathcal{O}=\{\langle F, D\rangle \mid F \subseteq \mathcal{F} \mathcal{T} \wedge D \subseteq \mathcal{T} \mathcal{T} \mathcal{F} \wedge D \subseteq T(F)\}
$$

For $o b=\langle F, D\rangle \in \mathcal{O}$ we define: $F(o b)=F, D(o b)=D$ and $T(o b)=T(F(o b))$.

\footnotetext{
${ }^{4}$ Brookes proved in [Bro83] that the identification of divergence with chaos is necessary for the sake of the continuity of the hiding operator in failure semantics, due to the instantaneous nature of its actions. As our actions are not instantaneous, it is possible, as we will see later, to define a sound semantics for recursive guarded processes, without such restrictive technicalities.
} 


\subsection{Specifications}

The notion of specification [0H86,Old86] is useful for the design and development of concurrent programs. We start with a specification $S p$ which denotes a set of behavioural observations that we expect in the behaviour of the program we are looking for. We say a program $P$ is correct with respect to $S p$ ( $P$ sat $S p)$, if all the behavioural observations of $P$ are within $S p$.

In our model specifications are timed observations $(S p \in \mathcal{O})$ with some restrictions. To each program $P$ we associate a specification $\mathcal{S} \llbracket P \rrbracket$, so that $P$ sat $S p$ when $S p \leq_{\mathrm{S}} \mathcal{S}[P]$, being $\leq$ s some partial order over $\mathcal{O}$.

Def. 2.9 We define the specification space: $\langle\mathcal{S}, \leq s\rangle$, where the set of specifications is $\mathcal{S}=\{o b \in \mathcal{O} \mid o b$ verifies [P1] - [P8] $\}$ is and the partial order over timed observations is $o b_{1} \leq_{\mathrm{s}} o b_{2} \stackrel{\text { def }}{=} F\left(o b_{2}\right) \subseteq F\left(o b_{1}\right) \wedge D\left(o b_{2}\right) \subseteq D\left(o b_{1}\right)$, for $o b_{1}, o b_{2} \in \mathcal{O}$.

- $[\mathrm{P} 1]\left(t_{\theta}, 0\right) \in T(o b)$

- [P2] $\left(t_{1}, n_{1}\right) \cdot\left(t_{2}, n_{2}\right) \in T(S p) \Rightarrow\left(t_{1}, n_{1}\right) \in T(S p)$

- [P3] $(t, n) \in T(o b) \wedge r \in \mathcal{R} \Rightarrow$ $(\exists B \in r:(t, n)+\mathrm{TB}(B, n+1) \in T(o b)) \vee\langle(t, n), r\rangle \in F(o b)$

- [P4] $\langle t f, r\rangle \in F(o b) \wedge r^{\prime} \subset r \Rightarrow\left\langle t f, r^{\prime}\right\rangle \in F(o b)$

- [P5] $t f \in D(o b) \Rightarrow \forall m \geq \sup (t f): t f[m \in D(o b)$

- [P6] $\langle t f, r\rangle \in F(o b) \wedge t f^{\prime} \in \operatorname{Stretch}(t f) \Rightarrow\left\langle t f^{\prime}, r\right\rangle \in F(o b)$

- [P7] $t f \in D(o b) \wedge t f^{\prime} \in \operatorname{Stretch}(t f) \Rightarrow t f^{\prime} \in D(o b)$

- [P8] $B \leq_{\mathrm{B}} B^{\prime} \wedge\langle t f, r \cup\{B\}\rangle \in F(o b) \Rightarrow\left\langle t f, r \cup\left\{B, B^{\prime}\right\}\right\rangle \in F(o b)$

Props. 2.1 $\langle\mathcal{S}, \leq s\rangle$ is a Complete Partial Order $(C P O)$.

\section{Semantics of TCSP-processes}

In the previous section we have only given a specification space suitable for defining denotational semantics, but we still must provide, for each syntactic operator in the programming language, a continuous operator over this specification space.

We consider the following set of syntactic operators for TCSP-processes:

$$
\Sigma_{1}=\{\text { stop }\} \cup\{a \rightarrow \mid a \in \mathcal{A}\} \cup\{\Pi, \square\} \cup\left\{\|_{A} \mid A \subseteq \mathcal{A}\right\} \cup\{\backslash a \mid a \in \mathcal{A}\}
$$

Def. 3.1 $A$ TCSP-process is a recursive term over $\Sigma_{1}\left(P \in R E C\left(\Sigma_{1}\right)\right)$ :

$$
P::=\text { stop }|a \rightarrow P| P \sqcap P|P \square P| P \|_{A} P|P \backslash a| \xi \mid \mu \xi . P
$$

where $\xi$ belongs to some process identifier set Id. 
Only recursive closed processes $C R E C\left(\Sigma_{1}\right)$ (without free identifiers) are considered.

We now give the semantic operator associated to each syntactic operator. The resulting specification has two components, the first one expressing the timed failures and the second, the divergences. Let $S p, S p_{1}, S p_{2} \in \mathcal{S}$,

1. $\mathcal{S}_{\text {stop }}\left[\cdot \mathbb{I}=\left\{\begin{array}{l}\left\{\left(\left(t_{\boldsymbol{\theta}}, m\right), r\right\rangle \mid m \in \mathbb{N} \wedge r \in \mathcal{R}\right\} \\ \emptyset\end{array}\right.\right.$

2. $\mathcal{S}_{a \rightarrow \llbracket S p \rrbracket}=\left\{\begin{array}{l}\left\{\left(\left(t_{\Phi}, m\right), r\right\rangle \mid m \in \mathbb{N} \wedge r \in \mathcal{P} \mathcal{F}\left(\mathcal{B}_{-a}^{+}\right)\right\} \\ \cup\left\{\left\langle\left(t_{n, a}, m\right), r\right\rangle \mid n \in \mathbb{N}^{+} \wedge n \leq m<n d \wedge r \in \mathcal{R}\right\} \\ \cup\left\{\left\langle\left(t_{n, a}, n d\right) \cdot t f, r\right\rangle \mid n \in \mathbb{N}^{+} \wedge(t f, r\rangle \in F(S p)\right\} \\ \\ \left\{\left(t_{n, a}, m\right) \mid n \in \mathbb{N}^{+} \wedge n \leq m<n d \wedge\left(t_{\emptyset}, 0\right) \in D(S p)\right\} \\ \cup\left\{\left(t_{n, a}, n d\right) \cdot t f \mid n \in \mathbb{N}^{+} \wedge t f \in D(S p)\right\}\end{array}\right.$

where $n d=n+d(a)-1$. Notice how action $a$ may start its execution at any instant. So long as $a$ does not start, any bag except from $B_{a}$ will be refused.

3. $\mathcal{S}_{\Pi}\left[S p_{1}, S p_{2}\right]=\left\{\begin{array}{l}F\left(S p_{1}\right) \cup F\left(S p_{2}\right) \\ D\left(S p_{1}\right) \cup D\left(S p_{2}\right)\end{array}\right.$

4. $\mathcal{S}_{\square}\left[S p_{1}, S p_{2} \rrbracket=\left\{\begin{array}{l}\left\{\left\langle\left(t_{\emptyset}, m\right), r\right\rangle \in F\left(S p_{1}\right) \cap F\left(S p_{2}\right)\right\} \\ \cup\left\{\langle t f, r\rangle \in F\left(S p_{1}\right) \cup F\left(S p_{2}\right) \mid t r a c e(t f) \neq t_{\oplus}\right\} \\ D\left(S p_{1}\right) \cup D\left(S p_{2}\right)\end{array}\right.\right.$

5. $\mathcal{S}_{\|_{A}}\left[S p_{1}, S p_{2}\right]=\left\{\begin{array}{c}\left\{\left\langle t f_{1} \oplus_{A} t f_{2}, r\right\rangle \mid\left\langle t f_{i}, r_{i}\right\rangle \in F\left(S p_{i}\right), i=1,2 \wedge r \in r_{1} \oplus_{A} r_{2}\right\} \\ \left\{t f_{1} \oplus_{A} t f_{2} \mid\left(t f_{1} \in D\left(S p_{1}\right) \wedge t f_{2} \in T\left(S p_{2}\right)\right)\right. \\ \left.\vee\left(t f_{1} \in T\left(S p_{1}\right) \wedge t f_{2} \in D\left(S p_{2}\right)\right)\right\}\end{array}\right.$

6. $\mathcal{S}_{\backslash a} \llbracket S p \rrbracket=\left\{\begin{aligned}\left\{\left(t f \backslash a, r \cup r^{\prime}\right\rangle \mid\langle t f, r\rangle \in F(S p) \wedge r^{\prime} \in \mathcal{P F}\left(\mathcal{B}_{a}\right)\right\} \\ \left\{t f \backslash a \mid \exists t f^{\prime}: \mathcal{A}\left(t f^{\prime}\right) \subseteq\{a\} \wedge t f \cdot t f^{\prime} \in D(S p)\right. \\ \left.\vee \exists\left\{t_{i}\right\}_{i \in \mathbb{N}^{+}}: \mathcal{A}\left(t_{i}\right)=\{a\} \wedge t f \cdot t_{1} \ldots t_{i} \in T(S p)\right\}\end{aligned}\right.$

Props. 3.1 $\forall o p \in \Sigma_{1}, \mathcal{S}_{o p} \llbracket S p_{1}, \ldots, S p_{n} \rrbracket$ is well defined $(\in \mathcal{O})$, monotonic, and it preserves properties $[P 1]-[P 8]$.

Props. 3.2 $\forall o p \in \Sigma_{1}-\{\backslash a\}, \mathcal{S}_{o p} \llbracket S p_{1}, \ldots, S p_{n} \rrbracket$ is continuous.

The hiding operator is not continuous on the whole specification space, due to the fact that we cannot distinguish between the execution of a unique hidden action and the simultaneous execution of several of them, as the time needed for it is the same (but this has nothing to do with the identification of divergence with chaos). 
Example 3.1 Let $\mathcal{A}=\{a, b\}$ with $d(a)=d(b)=1$. We define the process

$$
Q=a \rightarrow b \rightarrow \text { stop } \|_{\{b\}} Q
$$

This process can perform any amount of $a$ actions, but it never performs any $b$. Now we consider the process sequence $\left\{Q_{n}\right\}_{n \in \mathbb{N}}$ where

$$
\begin{aligned}
& Q_{0}=\operatorname{chaos}^{5} \\
& Q_{n}=a \rightarrow b \rightarrow \text { stop } \|_{\{b\}} Q_{n-1}
\end{aligned}
$$

Thus for each process $Q_{n-1}$ at least $\mathrm{n} a$ actions are executed before performing $b$.

If we define $X=\left\{\mathcal{S}\left[Q_{n}\right]\right\}_{n \in \mathbb{N}}$, then $X$ is a directed set and $\left.\operatorname{lub}(X)=\mathcal{S} \llbracket Q\right]$. However, when applying the hiding operator we obtain $\mathcal{S}_{\backslash a} \llbracket \operatorname{lub}(X) \rrbracket=\mathcal{S}_{\backslash a} \llbracket Q \rrbracket \neq \operatorname{lub}\left(\left\{\mathcal{S}_{\backslash a} \llbracket Q_{n} \rrbracket\right\}\right)$. For consider the trace $t f=\left(t_{2, b}, 2\right)$, then $\forall n \in \mathbb{N}: t f \in T\left(\mathcal{S}_{\backslash a} \llbracket Q_{n} \rrbracket\right)$ but $t f \notin T\left(\mathcal{S}_{\backslash a} \llbracket Q \rrbracket\right)$.

\subsection{Guarded processes}

If we restrict the dynamic process generation by requiring that every recursive call is preceded by the execution of at least one action, then in a bounded time interval we will obtain a bounded amount of parallel processes. We will call this class of processes guarded processes.

Def. 3.2 Well-defined expression $E$ :

$$
E::=\text { stop }|a \rightarrow E| E \sqcap E|E \square E| E \|_{A} E|E \backslash a| \xi \mid \mu \xi . P G
$$

Guarded process $P G \in R E C G\left(\Sigma_{1}\right)$ :

$$
P G::=\text { stop }|a \rightarrow E| P G \sqcap P G|P G \square P G| P G \|_{A} P G|P G \backslash a| \mu \xi . P G
$$

In distinction to untimed models, the hiding operator does not affect the guards in processes, as the guard is the duration of the action and not its name. This implies, in particular, that a guarded process may diverge. Take for instance: $(\mu \xi . a \rightarrow \xi \rightarrow$ stop $) \backslash a$.

The hiding operator is well-behaved (on the limit) when dealing with guarded processes. This is expressed by the following proposition:

Props. 3.3 Let $\sigma \in R E C G\left(\Sigma_{1}\right)$ be finite and with only one free variable. If $X_{\sigma}=$ $\left\{f_{\sigma}^{n}\left(\perp_{S}\right)\right\}_{n \geq 0}$, then $\operatorname{fix}\left(f_{\sigma}\right)=\operatorname{lub}\left(X_{\sigma}\right)$.

(If $\sigma \in R E C G\left(\Sigma_{1}\right)$ has only one free variable, then $\mathcal{S} \llbracket \sigma \rrbracket$ represents a function between specifications denoted by $f_{\sigma}: \mathcal{S} \longrightarrow \mathcal{S}$.)

Proof : the basic idea of the proof is that no recursive guarded process can perform infinitely many simultaneous actions (in a finite time). This is formalized in the following lemma:

\footnotetext{
${ }^{5}$ The semantics of chaos is the bottom of the specification space: $\perp_{s}=\langle\mathcal{F T}, \mathcal{T} \mathcal{T} \mathcal{F}\rangle$.
} 
Lemma 3.4 If $\sigma \in \operatorname{RECG}\left(\Sigma_{1}\right)$ is finite and with only one free variable, then for each $S p \in \mathcal{S}, \bigcup_{n \geq i} T\left(f_{\sigma}^{n}(S p)\right)$ is bounded in every time instant $i \in \mathbb{N}^{+}$:

$$
\forall i \in \mathbb{N}^{+} \exists k_{i}=\max \left\{|t(i)|, t \in \bigcup_{n \geq i} T\left(f_{\sigma}^{n}(S p)\right)\right\}
$$

with each iteration of the process we bound the size of the bags at one more instant.

\section{Proof System}

A denotational semantics defines the meaning of each process and induces a natural equivalence between processes: $P$ and $Q$ are equivalent iff they have the same semantics.

If we are really interested in this equivalence relation, we could establish it, at least in the finite cases, by computing the corresponding denotational semantics and comparing them. But it is clearly preferible to count on some indirect procedure which allows us to make such decisions without leaving the syntactic level in which processes are given. Thus we are interested in having some collection of algebraic laws which allows us to establish any valid equality. This is called a complete and correct proof system. Correct in the sense that every derived equality must be semantically valid, and complete in the sense that every semantically valid equality must be derivable by the system.

In order to prove that a system is correct it is sufficient to prove that every axiom and rule in the system is correct. Completeness of the system is more difficult to establish and the usual way is to define some normal form (each process can be transformed into a unique ${ }^{6}$ ) normal form which reflects as well as possible the nature of the semantics, so that two semantically equivalent processes have the same normal form.

This part of the work has been greatly inspired by [Hen88,Bro83,TV89]. The first presents a general theory; the second includes a proof system for a failure semantics for TCSP and the third for a failure semantics with multisets (bags). Complete proofs for every result presented in this section can be found in [Ort90].

We first concentrate on finite processes, i.e. closed processes without recursive calls in their syntactic definitions. Afterwards we extend the results to deal with recursion.

\subsection{Finite processes}

The main difficulty encountered when looking for the axiom system was defining an appropiate normal form. Following the steps of Taubner \& Vogler in [TV89] we introduce new and simpler operators, to be able to eliminate the more complex operators from the normal forms. Therefore, we will consider the following syntactic operator set:

$$
\Sigma_{x}=\Sigma_{1} \cup\left\{B \Rightarrow \mid B \in \mathcal{B}^{+}(\mathcal{A})\right\} \cup\{\text { delay, delay } *\}
$$

Def. 4.1 Extended TCSP term $P \in F I N\left(\Sigma_{x}\right)$ :

$$
P::=\text { stop }|a \rightarrow P| B \Rightarrow P|P \sqcap P| P \square P\left|P \|_{A}\right| P \backslash a|\operatorname{delay}(P)| \operatorname{delay} *(P)
$$

\footnotetext{
${ }^{6 r}$ To be accurate, unicity of normal forms is not required if the syntactic equivalence between the different normal forms corresponding to a same process can be easily established.
} 
The new prefix operator $B \Rightarrow$ is similar to $a \rightarrow$, but considering bags instead of single actions, allowing us to eliminate the synchronizing operator. It is assumed that the duration of bag $B$ is just one instant, thus a new operator delay is needed to "delay" the following process and to simulate longer durations. Process $\operatorname{delay}(P)$ waits one instant before starting the execution of $P$, thus representing both the execution of internal actions and the "passing of time". Notice how delay and $B \Rightarrow^{7}$ reflect the temporal nature of the semantics:

- $B \Rightarrow$ represents one time unit in which $B$ is observed,

- delay represents one time unit in which nothing is observed.

Moreover, $B$ (in $B \Rightarrow$ ) is executed immediately, so that a new operator, which expresses the idea of no maximum parallelism, is needed: $\operatorname{delay} *(P)$ may wait any amount of time before actually starting with the execution of $P$.

Now we are obliged to extend the semantic domain, because some of the new operators do not preserve properties [P1]-[P8]. The new domain is $\langle\mathcal{S} x, \leq \mathrm{s}\rangle$ :

$$
\begin{gathered}
\mathcal{S} x=\left\{o b \in \mathcal{O} \mid o b \text { verifies [P1] }-[\mathrm{P} 5],\left[\mathrm{P} 6^{\prime}\right]\right\} \\
{\left[\mathrm{P}^{\prime}\right](t, n) \in T(o b) \Rightarrow(t, n+1) \in T(o b)}
\end{gathered}
$$

Props. $4.1\langle\mathcal{S} x, \leq \mathrm{s}\rangle$ is a C.P.O.

Let $S p, S p_{1}, \ldots, S p_{n} \in \mathcal{S} x$, the corresponding semantic operators are:

- $\left.S x_{o p} \llbracket S p_{1}, \ldots, S p_{n}\right]=\mathcal{S}_{o p} \llbracket S p_{1}, \ldots, S p_{n} \rrbracket$ for $o p \in \Sigma_{1}-\{\backslash a\}$.

- $\mathcal{S} x_{B} \Rightarrow[S p]=\left\{\begin{array}{l}\left\{\left\langle\left(t_{\emptyset}, 0\right), r\right\rangle \mid r \in \mathcal{P} \mathcal{F}\left(\mathcal{B}^{+}-\{B\}\right)\right\} \cup\left\{\left(\left(t_{\theta}, m\right), r\right\rangle \mid m \in \mathbb{N}^{+} \wedge r \in \mathcal{R}\right\} \\ \cup\{\langle\operatorname{mov}(t f, 1)+\mathrm{TB}(B, 1), r\rangle \mid\langle t f, r\rangle \in F(S p)\} \\ \{\operatorname{mov}(t f, 1)+\mathrm{TB}(B, 1) \mid t f \in D(S p)\}\end{array}\right.$

If $B$ is not immediately executed (first instant) the process "loses its chance" of executing its first step and from then on, it cannot do anything but let the time pass and refuse every bag. This anomalous behaviour is introduced in order to preserve property [P6'], to make process stop be the unit of the external choice operator, and to get the distributivity between both choice operators.

- $\mathcal{S} x_{\text {delay }}[S p]=\left\{\begin{array}{l}\left\{\left\langle\left(t_{\emptyset}, 0\right), r\right\rangle \mid r \in \mathcal{R}\right\} \cup\{\langle\operatorname{mov}(t f, 1), r\rangle \mid\langle t f, r\rangle \in F(S p)\} \\ \left\{\left(t_{\emptyset}, 0\right) \in D(S p)\right\} \cup\{\operatorname{mov}(t f, 1) \mid t f \in D(S p)\}\end{array}\right.$

- $\mathcal{S}_{\text {delay* }}[S p]=\left\{\begin{array}{l}\left\{\left(\left(t_{\emptyset}, m\right), r\right\rangle \mid m \in \mathbb{N} \wedge \forall m^{\prime}=0 . . m,\left\langle\left(t_{\emptyset}, m^{\prime}\right), r\right\rangle \in F(S p)\right\} \\ \cup\left\{(\operatorname{mov}(t f, m), r\rangle \mid m \in \mathbb{N} \wedge \operatorname{trace}(t f) \neq t_{\emptyset} \wedge\langle t f, r\rangle \in F(S p)\right\} \\ \{\operatorname{mov}(t f, m) \mid m \in \mathbb{N} \wedge t f \in D(S p)\}\end{array}\right.$

Notice that $\operatorname{delay} *(P)$ represents a process which offers to the environment (external choice) the possibility of delaying the execution of $P$ as long as desired. Remember also that in external choice the decision is taken only when the first externally visible action starts. This idea is reflected on the first part of the semantical definition.

\footnotetext{
7The bag prefix operator is borrowed from [TV89]. A "delay" or "wait" operator appears in most timed models like in [RR87,MT89],etc.
} 


$$
\text { - } \left.S_{\backslash \backslash a} \llbracket S p\right]=\left\{\begin{array}{c}
\left\{\left\langle t f \backslash a, r \cup\left\{B_{1}, \ldots, B_{m}\right\}\right\rangle \mid r \in \mathcal{P} \mathcal{P}\left(\mathcal{B}_{a}\right)\right. \\
\left.\wedge \forall n_{1}, \ldots, n_{m} \in \mathbb{N}^{m}\left\langle t f, \bigcup_{i=1}^{m}\left\{\bigcup_{j=0}\left\{B_{i}+{ }_{\mathrm{B}} j * B_{a}\right\}\right\}\right\rangle \in F(S p)\right\} \\
\left\{t f \backslash a \mid \exists t f^{\prime}: \mathcal{A}\left(t f^{\prime}\right) \subseteq\{a\} \wedge t f \cdot t f^{\prime} \in D(S p)\right. \\
\left.\vee \exists\left\{t_{i}\right\}_{i \in \mathbb{N}^{+}}: \mathcal{A}\left(t_{i}\right)=\{a\} \wedge t f \cdot t_{\mathrm{r}} \ldots \cdot t_{\mathrm{i}} \in T(S p)\right\}
\end{array}\right.
$$

The hiding operator needs a small change due to the absence of property [P8]. The new definition coincides with the old one in the domain $\mathcal{S}$.

Props. 4.2 $\forall$ op $\in \Sigma_{x}, \mathcal{S} x_{o p}\left[S p_{1}, \ldots, S p_{n} \rrbracket\right.$ is well-defined, monotonic, and it preserves properties [P1]-[P5],[P6'].

Props. 4.3 $\forall o p \in \Sigma_{x}-\{\backslash a\}, \mathcal{S} x_{o p} \llbracket S p_{1}, \ldots, S p_{n} \rrbracket$ is continuous.

Again, as happened for $\left\langle\mathcal{S}, \leq_{s}\right\rangle$, the hiding operator is not continuous in general, but behaves well enough for guarded processes.

\subsubsection{Basic terms}

We consider a subset of TCSP extended terms:

Def. 4.2 Basic term $P \in T B A S$ :

$$
P::=\text { stop }|B \Rightarrow P| P \sqcap P|P \square P| \operatorname{delay}(P) \mid \operatorname{delay} *(P)
$$

We will prove that every TCSP extended term can be transformed into a basic term and every basic term into a normal form.

\subsubsection{Proof system}

The logic language for the proof system consists of extended TCSP terms plus a binary relation $\equiv .^{8}$

From now on we consider three different identity symbols:

- $=s$ represents semantical equality,

- = represents syntactical identity,

- $\equiv$ refers to the equivalence induced by the proof system.

By an abuse of notation we identify syntactic processes with its semantics, so that we drop $\mathcal{S} x[\cdot]$ when the context is obvious. For instance we write $P={ }_{S} Q$ for $\mathcal{S} x[P]=\mathcal{S} x[Q]$.

Tables 1 and 2 contain respectively the lists of axioms $\left(\Delta_{1}\right)$ and rules $\left(\Delta_{2}\right)$ of the proof system which allow for the transformation of any basic term into a normal form.

Axiom (D4) is like an "expansion" rule for the delay* operator. It expresses the fact that $\operatorname{delay} *(P)$ represents a process which offers the possibility of starting $P$ immediately or waiting one instant before choosing again. 

(A1) $P \sqcap P \equiv P$
(A2) $P \square P \equiv P$
(A3) $P \sqcap Q \equiv Q \sqcap P$
(A4) $P \square Q \equiv Q \square P$
(A5) $\quad P \sqcap(Q \sqcap R) \equiv(P \sqcap Q) \sqcap R$
(A6) $P \square(Q \square R) \equiv(P \square Q) \square R$
(A7) $\quad P \sqcap(Q \square R) \equiv(P \sqcap Q) \square(P \sqcap R)$
(A8) $P \square(Q \sqcap R) \equiv(P \square Q) \sqcap(P \square R)$
(A9) $P \square$ stop $\equiv P$
(A10) $\quad(B \Rightarrow P) \sqcap(B \Rightarrow Q) \equiv B \Rightarrow(P \sqcap Q)$
(A11) $(B \Rightarrow P) \square(B \Rightarrow Q) \equiv B \Rightarrow(P \sqcap Q)$
(D0) $\operatorname{delay}($ stop $) \equiv$ stop
(D0*) delay*(stop) $\equiv$ stop
(D1) $\operatorname{delay}(P \sqcap Q) \equiv \operatorname{delay}(P) \sqcap \operatorname{delay}(Q)$
(D2) $\quad \operatorname{delay}(P \square Q) \equiv \operatorname{delay}(P) \square \operatorname{delay}(Q)$
$(\mathrm{D} 2 *) \quad \operatorname{delay} *(P \square Q) \equiv \operatorname{delay} *(P) \square \operatorname{delay} *(Q)$
(D3) $\operatorname{delay}(\operatorname{delay} *(P)) \equiv \operatorname{delay} *(\operatorname{delay}(P))$

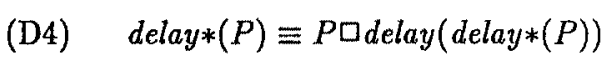
(D5) $\operatorname{delay} *(\operatorname{delay} *(P)) \equiv \operatorname{delay} *(P)$

Table 1: axiom set $\Delta_{1}$

\begin{tabular}{|c|c|c|c|}
\hline (01) & $P \equiv P$ & $(\mathrm{O} 2)$ & $\begin{array}{l}P \equiv Q \\
Q \equiv P\end{array}$ \\
\hline$(\mathrm{O} 3)$ & $\frac{P \equiv Q \wedge Q \equiv R}{P \equiv R}$ & (M1) & $\frac{P \equiv Q}{B \Rightarrow P \equiv B \Rightarrow Q}$ \\
\hline (M2) & $\frac{P_{1} \equiv Q_{1} \wedge P_{2} \equiv Q_{2}}{P_{1} \sqcap P_{2} \equiv Q_{1} \sqcap Q_{2}}$ & (M3) & $\frac{P_{1} \equiv Q_{1} \wedge P_{2} \equiv Q_{2}}{P_{1} \square P_{2} \equiv Q_{1} \square Q_{2}}$ \\
\hline (M4) & $\begin{array}{c}P \equiv Q \\
\operatorname{delay}(P) \equiv \operatorname{delay}(Q)\end{array}$ & $(\mathrm{M} 4 *)$ & 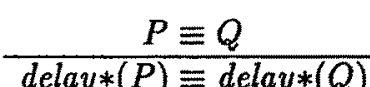 \\
\hline
\end{tabular}

Table 2: rule set $\Delta_{2}$ 
It would be nice to have also the following formula:

$$
(\mathrm{D} 1 *) \quad \operatorname{delay} *(P \sqcap Q) \equiv \operatorname{delay} *(P) \sqcap \operatorname{delay} *(Q)
$$

Unfortunately this is false in general. However, we will need at least some restricted form of it to complete our axiom system. Therefore, we.will investigate the grounds for its invalidity. First of all we have the following lemma:

Lemma 4.4 Let $\left.S p_{1}, S p_{2} \in \mathcal{S} x: \mathcal{S} x \llbracket \operatorname{delay} *\left(S p_{1} \sqcap S p_{2}\right)\right] \leq{ }_{\mathrm{S}} \mathcal{S} x\left[\operatorname{delay} *\left(S p_{1}\right) \sqcap \operatorname{delay} *\left(S p_{2}\right)\right]$.

Moreover, it is easy to check that the falsehood of $(\mathrm{D} 1 *)$ comes only from failures with empty traces. The conditions required to make it true are not trivial, and some previous concepts and definitions are needed. These will also be useful for the definition of normal forms.

\subsubsection{Derived operators}

The following derived operators are merely syntactic abbreviations. We define first two families of delay operators:

Def. 4.3 General delay operators $\operatorname{delay}(\ell)$ and delay $*(\ell)$ with $\ell \in \mathbb{N}$ :

$$
\begin{aligned}
& \operatorname{delay}(0, P)=P, \\
& \operatorname{delay}(\ell+1, P)=\operatorname{delay}(\ell, \operatorname{delay}(P)), \\
& \operatorname{delay} *(\ell, P)=\operatorname{delay}(\ell, \operatorname{delay} *(P)) .
\end{aligned}
$$

Notice that $\operatorname{delay}(P)=\operatorname{delay}(1, P)$ and $\operatorname{delay} *(P)=\operatorname{delay} *(0, P)$.

Thanks to axioms (A2), (A4), (A6) and (A9) we can define $\square_{i \in I} P_{i}$, where $I$ is any finite index set and $P_{i} \in \mathcal{S} x$ for $i \in I$ :

$$
\begin{aligned}
& \square_{i \in \mathbb{Q}} P_{i}=\text { stop, } \\
& \square_{i \in j \cup I} P_{i}=P_{j} \square\left(\square_{i \in I} P_{i}\right) .
\end{aligned}
$$

If $I$ were allowed to be infinite, we would not need the delay* operator, as we could represent it by $\square_{m \geq 0}$ delay $(m, P)$. But, as it is well-known, such infinite terms are unsuitable, in general, within the framework of axiomatisation of algebraic processes.

Analogously we define $\mathrm{OR}_{i \in I} P_{i}$, but now $I$ cannot be the empty set:

$$
\begin{aligned}
& \mathrm{OR}_{i \in\{j\}} P_{i}=P_{j}, \\
& \mathrm{OR}_{i \in j \cup I} P_{i}=P_{j} \sqcap\left(\mathrm{OR}_{i \in I} P_{i}\right) \text { if } I \neq \emptyset .
\end{aligned}
$$

\footnotetext{
${ }^{8}$ In [Bro83,Hen88,TV89] inequation systems are considered. While we restrict ourselves to finite processes, the results are in fact equivalent, but since it is possible, we consider more elegant to work only with equations. However when dealing with recursive processes inequations will be unavoidable.
} 


\subsubsection{Prenormal form}

As candidates for our normal form we are looking for syntactic formulae which express more clearly the internal structure (what is happening at each instant) of the processes denoted by them.

Def. 4.4 We associate action bags to time instants and basic terms:

timed bags : $\mathcal{B T}=\mathcal{B}(\mathcal{A}) \times \mathbb{N}$,

sets of timed bags : $\mathcal{C B T}=\mathcal{P} \mathcal{F}(\mathcal{B T})$,

bag-process pairs: $\mathcal{B P}=\mathcal{B}(\mathcal{A}) \times T B A S$,

sets of bag-process pairs : $\mathcal{C B P}=\mathcal{P F}(\mathcal{B P})$.

For each $B T \in \mathcal{C B T}$, the set of bags in $B T$ is denoted by $\mathcal{B}(B T)$ (analogously for $B P \in$ $\mathcal{C B P})$.

We will usually consider pairs of timed bag sets, where the first component refers to delay operators and the second to delay*.

Def. 4.5 For each $C=\left(C_{1}, C_{2}\right) \in \mathcal{C B T} \times \mathcal{C B T}$, we define:

timed bags in $C: \mathcal{B} T(C)=C_{1} \cup C_{2}$,

top time $: \operatorname{top}(C)= \begin{cases}-1 & \text { if } C=(\emptyset, \emptyset) \\ \max \left\{t \mid(B, t) \in C_{1} \vee(B, t+1) \in C_{2}\right\} & \text { otherwise }\end{cases}$

projection : $\Gamma_{j}(C)=C_{j}, j=1,2$.

As we will see, the top time spots the limit of changes in the activity of a process. From the top time on, the process will maintain the same options until the first non-empty action bag will be executed.

The above definitions can be generalized to sets of pairs:

Def. 4.6 Let $\mathrm{C}=\left\{C_{i}\right\}_{i=1 . . n} \in \mathcal{P F}(\mathcal{C B T} \times \mathcal{C B T})$, then we define:

timed bags in $C: \mathcal{B T}(\mathrm{C})=\bigcup_{i=1, \ldots} \mathcal{B} \mathcal{T}\left(C_{i}\right)$,

top time $: \operatorname{top}(\mathrm{C})=\max \left\{\operatorname{top}\left(C_{i}\right)\right\}_{i=1 . n \text {, }}$

projection : $\Gamma_{j}(\mathrm{C})=\bigcup_{1 \leq i \leq n} \Gamma_{j}\left(C_{i}\right), j=1,2$.

A first step towards the desired normal forms are prenormal forms : 
Def. 4.7 A term is in prenormal form if it is of form :

$$
\mathrm{OR}_{\left(C_{1}, C_{2}\right) \in \mathrm{C}}\left[\square_{(B, t) \in C_{1}} \operatorname{delay}\left(t, B \Rightarrow P_{(B, t)}\right) \square \square(B, t) \in C_{2} \operatorname{delay} *\left(t, B \Rightarrow P_{(B, t)}\right)\right]
$$

where $\mathrm{C} \in \mathcal{P F}(\mathcal{C B T} \times \mathcal{C B T})$ and each $P_{(B, t)}$ is in prenormal form.

$A$ prenormal form is said unitary, when $\mathrm{C}$ consists of a unique pair of timed bag sets.

Notice that stop is in prenormal form.

Subprocesses $P_{(B, t)}$ depend only on $B$ and $t$, but not on $\left(C_{1}, C_{2}\right)$. Therefore, a prenormal form is completely defined by the pair $\langle\mathrm{C}, f p\rangle$, where $f p$ is a partial function which associates a basic term to each timed bag:

$$
f p(B, t)= \begin{cases}P_{(B, t)} & \text { if }(B, t) \in \mathcal{B T}(\mathrm{C}) \\ \perp & \text { otherwise }\end{cases}
$$

\subsubsection{Expansion and distribution}

Prenormal forms are too general to work with, because too many different prenormal forms may correspond to the same process. Moreover, the combination of prenormal forms with the internal choice operator not always can be transformed to another prenormal form. In order to overcome these difficulties, we will define the concepts of expansion and distribution.

Def. 4.8 Let $\langle C, f p\rangle$ be a unitary prenormal form with $C=\left\{\left(C_{1}, C_{2}\right)\right\}$ and $\operatorname{top}(C)=$ $K$. For every $m \geq K$, a set of $m+2$-tuples $\operatorname{EX}(\langle C, f p\rangle, m)$ is defined which is called expansion of $\langle C, f p\rangle$ until instant $\mathrm{m}$ :

$$
\operatorname{EX}(\langle C, f p\rangle, m)=\langle D(0), \ldots, D(m+1)\rangle \in \mathcal{P F}(\mathcal{C B P} \times \ldots \times \mathcal{C B P})(m+1 \text { times })
$$

where $D(j)=\left\{(B, f p(B, j)) \mid(B, j) \in C_{1}\right\} \cup\left\{(B, f p(B, t)) \mid t \leq j \wedge(B, t) \in C_{2}\right\}$.

When $m=K$ we write $\operatorname{EX}(\langle C, f p\rangle)$.

Expanding until instant $m$ means developing the $\operatorname{delay} *(\ell)$ operators on $\operatorname{delay}(j)$ operators for every $j: \ell \leq j \leq m$ plus delay*(m+1). Thus $C$ is decomposed in timing groups $D(t), t=0 . . m+1$.

Def. 4.9 Let $\langle\mathrm{C}, f p\rangle$ be a prenormal form with $K=\operatorname{top}(\mathrm{C})$. Two families of tuple sets are defined:

Expansion : $\forall m \geq K, \operatorname{EX}(\langle\mathrm{C}, f p\rangle, m)=\{\operatorname{EX}(\langle C, f p\rangle, m)\}_{C \in \mathrm{C}}$.

When $m=K$ we write $\mathrm{EX}(\langle\mathrm{C}, f p\rangle)$.

Distribution : $\forall m=0 . . K+1, \operatorname{DSTR}(\langle\mathrm{C}, f p\rangle, m) \in \mathcal{P} \mathcal{F}(\mathcal{C B P} \times \ldots \times \mathcal{C B P})(K+2$ times $)$,

$\operatorname{DSTR}(\langle\mathrm{C}, f p\rangle, m)=\left\{D_{C}(0)\right\}_{C \in \mathrm{C}^{\times}} \ldots \times\left\{D_{C}(m-1)\right\}_{C \in} \mathrm{C}^{\times\left\{\left\langle D_{C}(m), \ldots, D_{C}(K+1)\right\rangle\right\}_{C \epsilon}}$ where $\operatorname{EX}(\langle C, f p\rangle)=\left\langle D_{C}(0), \ldots, D_{C}(K+1)\right\rangle$ for each $C \in \mathrm{C}$.

When $m=K+1$ we write $\operatorname{DSTR}(\langle\mathrm{C}, f p\rangle)$. 
if $\langle\mathrm{C}, f p\rangle$ is a distributed prenormal form.

$(\mathrm{D} 1 *) \quad \operatorname{delay} *(\langle\mathrm{C}, f p\rangle) \equiv \mathrm{OR}_{C \in \mathrm{C}} \mathrm{C}^{\operatorname{delay} *(\langle C, f p\rangle)}$

Let $R=\left(P_{1} \square \operatorname{delay}\left(k, Q_{1}\right)\right) \Pi\left(P_{2} \square \operatorname{delay}\left(k, Q_{2}\right)\right)$

with $P_{i}=\square_{0 \leq j<k} \square_{(B, P) \in c_{i j}} \operatorname{delay}(j, B \Rightarrow P)$

(D6)

$$
R \equiv R \sqcap\left(P_{1} \square \operatorname{delay}\left(k, Q_{2}\right)\right) \sqcap\left(P_{2} \square \operatorname{delay}\left(k, Q_{1}\right)\right)
$$

Table 3: distribution axiom and law

Distributing until instant $m$ means obtaining every combination of timing groups until instant $m$. If $\operatorname{top}(\mathrm{C})=K$ we build tuples $\langle D(0), \ldots, D(K+1)\rangle$ where each $D(i), 0 \leq i<$ $m$ belongs to some element of $\mathrm{C}$, maybe different each time, but every $D(i), m \leq i \leq K+1$ belongs to the same element. Notice that $\operatorname{DSTR}(\langle\mathrm{C}, f p\rangle, 0)=\operatorname{EX}(\langle\mathrm{C}, f p\rangle)$.

Def. 4.10 A prenormal form $\langle\mathrm{C}, f p\rangle$ is said to be

expanded until instant $m(m \geq 0)$ if $\operatorname{top}(\mathrm{C})=m$, and $(B, t) \in \Gamma_{2}(\mathrm{C}) \Longrightarrow t=m+1$.

When $m=\operatorname{top}(\mathrm{C})$ we say expanded.

distributed until instant $\mathbf{m}(0 \leq m \leq t o p(\mathrm{C})+1)$ if $\operatorname{DSTR}(\langle\mathrm{C}, f p\rangle, m)=\operatorname{EX}(\langle\mathrm{C}, f p\rangle)$.

When $m=\operatorname{top}(\mathrm{C})+1$ we say distributed.

We are now prepared to give the axiom corresponding to the distribution of the delay* operator with the internal choice, and which should be included in the list $\Delta_{1}$ of table 1 . We also include in $\Delta_{1}$ the distribution law, a family of axiom which allow for the transformation of any prenormal form into a distributed one. These axioms appear on table 3 .

From now on we write $\vdash P \equiv Q$, when the formula $P \equiv Q$ is provable in $\Delta_{1} \cup \Delta_{2}$.

Theorem 4.5 For every $P, Q, R, \ldots$ TCSP extended terms, the axioms and rules in $\Delta_{1} U$ $\Delta_{2}$ are correct; i.e. $\vdash P \equiv Q$ implies $P=s Q$. 


\subsubsection{Normal form}

A normal form is a prenormal form verifying some conditions.

Def. 4.11 $\mathrm{C}$ is convex if it is non-empty and verifies:

1. $A, B \in \mathrm{C} \Longrightarrow A \cup B \in \mathrm{C}$,

2. $A, D \in \mathrm{C} \wedge A \subseteq B \subseteq D \Longrightarrow B \in \mathrm{C}$.

Def. 4.12 $A$ term is in normal form if it is in distributed prenormal form $\langle\mathrm{C}, f p\rangle$ with $\mathrm{C}$ convex and every $f p(B, t)$ in normal form.

We will give next some lemmas and propositions to transform any prenormal form into a normal one. First lemma states the convexity laws, already presented in [Hen88]:

Lemma 4.6 The following formulas are provable in $\Delta_{1} \cup \Delta_{2}$ :

$$
\begin{aligned}
& \text { (DV1) } P \sqcap Q \equiv P \sqcap Q \sqcap(P \square Q) \\
& \text { (DV2) } P \sqcap(P \square Q \square R) \equiv P \sqcap(P \square Q) \cap(P \square Q \square R)
\end{aligned}
$$

Next lemma states that in any prenormal form, $\mathrm{C}$ can be replaced by its convex closure $(\mathrm{CONV}(\mathrm{C}))$.

Lemma 4.7 $\vdash\langle\mathrm{C}, f p\rangle \equiv\langle\operatorname{CONV}(\mathrm{C}), f p\rangle$.

Proof : We apply the convexity laws (lemma 4.6).

Lemma 4.8 Let $P=P_{1} \sqcap P_{2}$, the following formulas are provable in $\Delta_{1} \cup \Delta_{2}$ :

(DV3) $\quad\left(\right.$ delay $\left.\left(\ell, B \Rightarrow P_{1}\right) \square Q_{1}\right) \sqcap\left(\operatorname{delay}\left(\ell, B \Rightarrow P_{2}\right) \square Q_{2}\right) \equiv$ $\left(\operatorname{delay}(\ell, B \Rightarrow P) \square Q_{1}\right) \sqcap\left(\operatorname{delay}(\ell, B \Rightarrow P) \square Q_{2}\right)$

$(\mathrm{DV} 3 *) \quad\left(\right.$ delay $\left.*\left(\ell, B \Rightarrow P_{1}\right) \square Q_{1}\right) \sqcap\left(\right.$ delay $\left.*\left(\ell, B \Rightarrow P_{2}\right) \square Q_{2}\right) \equiv$ $\left(\right.$ delay $\left.*(\ell, B \Rightarrow P) \square Q_{1}\right) \sqcap\left(\right.$ delay $\left.*(\ell, B \Rightarrow P) \square Q_{2}\right)$

The above rules are called expansion laws and are used to transform any prenormal form into an expanded one.

Lemma 4.9 Let $\langle\mathrm{C}, f p\rangle$ be a prenormal form,

1. $\forall m \geq \operatorname{top}(\mathrm{C}), \exists\left\langle\mathrm{C}^{\prime}, f p^{\prime}\right\rangle$ expanded until instant $m$ such as $\vdash\langle\mathrm{C}, f p\rangle \equiv\left\langle\mathrm{C}^{\prime}, f p^{\prime}\right\rangle$,

2. $\forall m=0 . . t o p(\mathrm{C})+1, \exists\left\langle\mathrm{C}^{\prime}, f p^{\prime}\right\rangle$ distributed until instant $m$ and expanded, such as $\operatorname{top}\left(\left\langle\mathrm{C}^{\prime}, f p^{\prime}\right\rangle=\operatorname{top}(\langle\mathrm{C}, f p\rangle)\right.$ and $\vdash\langle\mathrm{C}, f p\rangle \equiv\left\langle\mathrm{C}^{\prime}, f p^{\prime}\right\rangle$.

We also must guarantee that the convexity closure preserves distribution. 
Lemma 4.10 If $\langle\mathrm{C}, f p\rangle$ is a distributed prenormal form, then $\langle\mathrm{CONV}(\mathrm{C}), f p\rangle$ is distributed too.

A corollary of lemmas $4.9,4.7$ and 4.10 is given next:

Corollary 4.11 Let $\langle\mathrm{C}, f p\rangle$ be a prenormal form, there exists a normal form $\left\langle\mathrm{C}^{\prime}, f p^{\prime}\right\rangle$ such as $\vdash\langle\mathrm{C}, f p\rangle \equiv\left\langle\mathrm{C}^{\prime}, f p^{\prime}\right\rangle$.

We can finally state the expected proposition:

Props. 4.12 For each basic term $P$ there exists a normal form $\langle\mathrm{C}, f p\rangle$ such as $\vdash P \equiv$ $\langle\mathrm{C}, f p\rangle$.

\subsubsection{Proof system completeness}

In order to prove that the system is complete, we need to prove first that two semantically equivalent normal forms are syntactically equivalent. ${ }^{9}$

Props. 4.13 Let $\langle\mathrm{C}, f p\rangle$ and $\left\langle\mathrm{C}^{\prime}, f p^{\prime}\right\rangle$ be normal forms, then $\langle\mathrm{C}, f p\rangle=s\left\langle\mathrm{C}^{\prime}, f p^{\prime}\right\rangle$ implies $\vdash\langle\mathrm{C}, f p\rangle \equiv\left\langle\mathrm{C}^{\prime}, f p^{\prime}\right\rangle$.

Combining propositions 4.12 and 4.13 , we obtain the following theorem:

Theorem 4.14 Let $P, Q$ be basic terms, then $P={ }_{s} Q$ implies $\vdash P \equiv Q$.

\subsubsection{Transformation of finite TCSP terms into basic terms}

The table 4 contains the axiom list $\Delta_{3}$, which added to $\Delta_{1} \cup \Delta_{2}$ allows for the transformation of any finite TCSP term into a basic one. We obtain then a proof system for finite TCSP processes, which is correct and complete with respect to the denotational semantics given in section 3 . The more complex axioms are a consequence of the non-distributivity of the synchronization and hiding operators with the external choice.

Theorem 4.15 For any extended TCSP term $P, Q, R, \ldots$ and $C, D \in \mathcal{R}$ the axioms in $\Delta_{3}$ are correct.

Props. 4.16 Any extended TCSP term can be transformed, by means of the axioms and rules in $\Delta_{1}, \Delta_{2}$ and $\Delta_{3}$ into a basic term.

We shall consider from now on that $\vdash P \equiv Q$ means that the formula $P \equiv Q$ is provable in $\Delta_{1} \cup \Delta_{2} \cup \Delta_{3}$. We give next the last theorem of this part devoted to finite processes, which is a direct consequence of proposition 4.16 and theorems $4.5,4.14$ and 4.15 .

Theorem 4.17 Let $P, Q$ be finite TCSP terms, then $P={ }_{S} Q \Leftrightarrow \vdash P \equiv Q$.

\footnotetext{
${ }^{9}$ We cannot assert that they are syntactically identical, due to the fact that our normal forms are not unique. We could have defined some order over the set of normal forms for a process, obtaining a unique least normal form, but this is unnecessary.
} 
(X) $a \rightarrow P \equiv \operatorname{delay} *\left(0, B_{a} \Rightarrow \operatorname{delay}(d(a)-1, P)\right)$

(S0) stop $\|_{A}$ stop $\equiv$ stop

(S1) $P\left\|_{A} Q \equiv Q\right\|_{A} P$

(S2) $P \|_{A}(Q \sqcap R) \equiv\left(P \|_{A} Q\right) \sqcap\left(P \|_{A} R\right)$

(S3) $\operatorname{delay}(P) \|_{A} \operatorname{delay}(Q) \equiv \operatorname{delay}\left(P \|_{A} Q\right)$

(S4) Let $P=\left[\square_{B \in C} B \Rightarrow P_{B}\right] \square \operatorname{delay}\left(P^{\prime}\right)$ and $Q=\left[\square_{E \in D} E \Rightarrow Q_{E}\right] \square \operatorname{delay}\left(Q^{\prime}\right)$

$$
\begin{aligned}
P \|_{A} Q \equiv & \square \\
\square & \square_{B\lceil A=E\lceil A}\left(B \oplus_{A} E\right) \Rightarrow\left(P_{B} \|_{A} Q_{E}\right) \\
& \square \\
& \square_{B\left\lceil A=B_{\emptyset}\right.} B \Rightarrow\left(P_{B} \|_{A} Q^{\prime}\right) \\
& \square \\
& \square_{E\left[A=B_{\mathfrak{\vartheta}}\right.} E \Rightarrow\left(P^{\prime} \|_{A} Q_{E}\right) \\
& \operatorname{delay}\left(P^{\prime} \|_{A} Q^{\prime}\right)
\end{aligned}
$$

(S5) Let $P=\operatorname{delay} *\left(\square_{B \in C} B \Rightarrow P_{B}\right)$ and $Q=\operatorname{delay} *\left(\square_{E \in D} E \Rightarrow Q_{E}\right)$, then

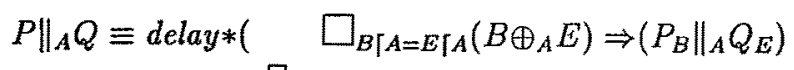

$$
\begin{gathered}
\square \\
\square\left[\begin{array}{c}
\square=B_{\emptyset} \\
\square
\end{array} \square_{E\left[A=B_{\emptyset}\right.} E \Rightarrow\left(P P_{B} \|_{A} Q\right)\right. \\
\left.\left.\square_{A} Q_{E}\right)\right)
\end{gathered}
$$

(H0) stop $\backslash a \equiv$ stop

(H1) $(P \sqcap Q) \backslash a \equiv P \backslash a \sqcap Q \backslash a$

(H2) $\operatorname{delay}(P) \backslash a \equiv \operatorname{delay}(P \backslash a)$

(H3) Let $C \cap \mathcal{B}_{a}=\emptyset$, then

$\left[\square_{B \in C} B \Rightarrow P_{B} \square \operatorname{delay}(P)\right] \backslash a \equiv\left[\square_{B \in C} B \backslash a \Rightarrow P_{B} \backslash a\right] \square \operatorname{delay}(P) \backslash a$

(H4) Let $C \cap \mathcal{B}_{a} \neq \emptyset$, then

$$
\left[\square_{B \in C} B \Rightarrow P_{B} \square \operatorname{delay}(P)\right] \backslash a \equiv\left[\square_{B \in C-\mathcal{B}_{a}} B \backslash a \Rightarrow P_{B} \backslash a\right] \square \operatorname{delay}\left(P \sqcap \mathrm{OR}_{B \in C \cap \mathcal{B}_{a}} P_{B}\right) \backslash a
$$

(H5) Let $C \cap \mathcal{B}_{a}=\emptyset$, then

$$
\text { delay } *\left(\square_{B \in C} B \Rightarrow P_{B}\right) \backslash a \equiv \text { delay } *\left(\square_{B \in C} B \backslash a \Rightarrow P_{B} \backslash a\right)
$$

(H6) Let $C \cap \mathcal{B}_{a} \neq \emptyset$, then

$$
\begin{aligned}
\operatorname{delay} *\left(\square_{B \in C} B \Rightarrow P_{B}\right) \backslash a \equiv & \square_{B \in C-\mathcal{B}_{a}} B \backslash a \Rightarrow P_{B} \backslash a \square \\
& \operatorname{delay}\left(\text { delay } *\left(\left[\square_{B \in C-\mathcal{B}_{a}} B \backslash a \Rightarrow P_{B} \backslash a\right] \cap O R_{B \in C \cap \mathcal{B}_{a}} P_{B} \backslash a\right)\right)
\end{aligned}
$$

Table 4: transformation axiom set $\Delta_{3}$ 


\subsection{Recursive processes}

In order to deal with recursive processes we need their syntactic aproximations corresponding to the semantic aproximations deduced from the fix-point theory. So as to work with aproximations, we need to introduce some changes in the proof system above given for finite processes. For one instance, it is no longer sufficient to deal with equivalences, and some partial order between processes is needed. Consequentely a new symbol is introduced (ㄷ), from which the syntactic equivalence () can be deduced. Another addition is the syntactic equivalent to the least element of the semantic domain: the process constant chaos.

\subsubsection{Superextended TCSP}

The the new set of syntactic operators is $\Sigma_{X}=\Sigma_{x} \cup\{$ chaos\}. We can maintain the same

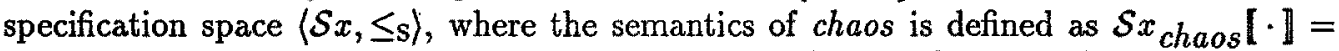
$\langle\mathcal{F} \mathcal{T}, \mathcal{T} \mathcal{T} \mathcal{F}\rangle$ (which is obviously well-defined, verifies [P1]-[P5] and [P6'], and is continuous).

\subsubsection{Syntactic aproximations}

The semantics of a recursive process $P$ is the limit of the sequence generated by unwinding the recursive calls, and starting with chaos:

$$
\begin{aligned}
& P^{o}=\text { chaos, } \\
& P^{n+1}=P\left[P^{n}\right] .
\end{aligned}
$$

Syntactically each $P^{n}$ is a superextended TCSP term $\left(P^{n} \in F I N\left(\Sigma_{X}\right)\right)$.

Def. 4.13 The set of finite aproximations for process $P$ is $\operatorname{APX}(P)=\left\{P^{n} \mid n \in \mathbb{N}\right\}$.

\subsubsection{New axioms and rules}

We must include, on the one hand, the axioms concerning the behaviour of chaos with respect to the other operators $\left(\Omega_{1}\right)$; on the other hand, the rules concerning recursion $\left(\Omega_{2}\right)$; and finally the rules justifying that $\sqsubseteq$ is a partial order $\left(\Omega_{3}\right)$. The tables 5,6 and 7 contain these sets respectively. Notice that the rules in $\Delta_{2}$ can be obtained from the rules in $\Omega_{2}$. We shall write $\vdash_{x} P \sqsubseteq Q$ when the formula $P \sqsubseteq Q$ is provable in $\Delta_{1} \cup \Omega_{1} \cup \Omega_{2} \cup \Omega_{3}$.

\subsubsection{Correctness of the new proof system}

First of all we must confess that the new system is not completely correct, as axioms (CH2), (CH4) and (CH5) are not correct. However "don't panic". As we are only interested in dealing with chaos in relation to the finite aproximations for recursive guarded processes, the inclusion of these "incorrect" axioms is justified by the fact that $P \square$ chaos, $P \|_{A}$ chaos and chaos $\backslash a$ have such a "chaotic" behaviour that it is feasible to identify them with chaos when they appear in the aproximations. We shall formalize the concept of partial correctness, and we will see that by means of this concept we can prove the total correctness of the system restricted to guarded TCSP processes. The rest of axioms and rules are correct. 


$\begin{array}{ll}\text { (A12) } & P \sqcap Q \sqsubseteq P \\ \text { (CH0) } & \text { chaos } \sqsubseteq P \\ \text { (CH1) } & P \sqcap \text { chaos } \equiv \text { chaos } \\ \text { (CH2) } & P \square c h a o s \equiv \text { chaos } \\ \text { (CH3) } & \text { delay } *(\text { chaos }) \equiv \text { chaos } \\ \text { (CH4) } & \text { chaos } \|_{A} P \equiv \text { chaos } \\ \text { (CH5) } & \text { chaos } \backslash a \equiv \text { chaos }\end{array}$

Table 5: axiom set $\Omega_{1}$

$$
\begin{aligned}
& \text { (01') } \frac{P \sqsubseteq Q \sqsubseteq P}{P \equiv Q} \\
& \text { (O2') } \frac{P \equiv Q}{P \sqsubseteq Q \sqsubseteq P} \\
& \text { (O3') } \frac{P \sqsubseteq Q \sqsubseteq R}{P \sqsubseteq R} \\
& \text { (M1') } \frac{P \sqsubseteq Q}{B \Rightarrow P \sqsubseteq B \Rightarrow Q} \\
& \left(\mathrm{M}^{\prime}\right) \quad \frac{P_{1} \sqsubseteq Q_{1} \wedge P_{2} \sqsubseteq Q_{2}}{P_{3} \sqcap P_{2} \sqsubseteq Q_{1} \sqcap Q_{2}} \\
& \text { (M3') } \frac{P_{1} \sqsubseteq Q_{1} \wedge P_{2} \sqsubseteq Q_{2}}{P_{1} \square P_{2} \sqsubseteq Q_{1} \square Q_{2}} \\
& \left(\mathrm{M} 4^{\prime}\right) \quad \frac{P \sqsubseteq Q}{\operatorname{delay}(P) \sqsubseteq \operatorname{delay}(Q)} \\
& \left(\mathrm{M} 4 *^{\prime}\right) \quad \frac{P \sqsubseteq Q}{\operatorname{delay} *(P) \sqsubseteq \operatorname{delay} *(Q)}
\end{aligned}
$$

Table 6: rule set $\Omega_{2}$

(R1) $P[\mu \xi . P / \xi] \sqsubseteq \mu \xi . P$

(R2) $\frac{\forall Q \in \operatorname{APX}(P): Q \sqsubseteq R}{P \sqsubseteq R}$

Table 7: recursion rules $\Omega_{3}$ 
Lemma 4.18 Let $P, Q, R, \ldots$ be extended and guarded TCSP terms, then the axioms in $\Omega_{2} \cup \Omega_{3} \cup\{(C H 0),(C H 1),(C H 4)\}$ are correct.

A family of semantic orders, based on the length of the observed traces, is introduced.

Def. 4.14 Let $S p \in \mathcal{S} x$ and $n \in \mathbb{N}$, we define:

$$
\begin{aligned}
& F_{n}(S p)=\{\langle t f, \emptyset\rangle \in F(S p) \mid e n d(t f)=n\} \cup\{\langle t f, r\rangle \in F(S p) \mid e n d(t f)<n\} \\
& D_{n}(S p)=\{t f \in D(S p) \mid e n d(t f) \leq n\} \\
& O B_{n}(S p)=\left\langle F_{n}(S p), D_{n}(S p)\right\rangle .
\end{aligned}
$$

Def. 4.15 Let $S p_{1}, S p_{2} \in \mathcal{S} x$ and $n \in \mathbb{N}: S p_{1} \leq_{n} S p_{2} \Leftrightarrow O B_{n}\left(S p_{2}\right) \subseteq O B_{n}\left(S p_{1}\right)$.

We shall write $P \leq_{n} Q$ when $\left.\mathcal{S} x \llbracket P \rrbracket \leq_{n} \mathcal{S} x \llbracket Q\right]$. These orders are only partial orders which induce the corresponding equivalences ${ }_{n}{ }_{n}$ between processes.

The relation between these new orders and the partial order $\leq \mathrm{s}$ is given by the following lemma:

Lemma 4.19 Let $S p_{1}, S p_{2} \in \mathcal{S} x$, then $S p_{1} \leq_{S} S p_{2} \Leftrightarrow \forall n \in \mathbb{N}: S p_{1} \leq_{n} S p_{2}$.

Lemma 4.20 Let $P \in C R E C G\left(\Sigma_{1}\right)$, then $\forall n \in \mathbb{N}: P={ }_{n} P^{n}$.

Lemma 4.21 (Partial correctness) Let $P, Q \in C R E C G\left(\Sigma_{1}\right): P^{n} \sqsubseteq Q \Longrightarrow P^{n} \leq_{n} Q$. Proof : As $P$ is guarded it is easily proven that each occurence of chaos in $P^{n}$ is guarded $\mathrm{n}$ times. The application of any axiom or rule in our present proof system preserves this property. The application of correct axioms preserves $\leq_{\mathrm{S}}$, and consequently each $\leq_{n}$ too. Whereas the application of "incorrect" axioms preserves $\leq_{n}$ too, because thay are only applied under $\mathbf{n}$ guards.

We can now prove the correctness with respect to guarded processes.

Props. 4.22 Let $P, Q \in C R E C G\left(\Sigma_{1}\right)$, then $\vdash_{x} P \sqsubseteq Q$ implies $P \leq{ }_{\mathrm{S}} Q$.

Proof : For every $P^{n} \in \operatorname{APX}(P)$ it is proved that $P^{n} \sqsubseteq P \sqsubseteq Q$. The partial correctness lemma 4.21 is applied and we obtain $P^{n} \leq_{n} Q$. Therefore, by lemma 4.20 we obtain that $\forall n \in \mathbb{N}: P \leq_{n} Q$. We conclude thanks to lemma 4.19.

\subsubsection{Completeness of the new system}

We shall start with the completeness with respect to finite terms. We only need to "extend" all the results obtained before for finite TCSP terms, to extended TCSP terms. For we introduce chaos in the normal forms.

Def. 4.16 Superbasic term $P \in S T B A S$ :

$$
P::=\text { chaos } \mid \text { stop }|B \Rightarrow P| P \sqcap P|P \square P| \operatorname{delay}(P) \mid \operatorname{delay} *(P)
$$


Def. 4.17 A term is in superprenormal form if it is of any of the following forms:

- $\langle\mathrm{C}, f p\rangle$ prenormal form with each $f p(B, t)$ in superprenormal form,

- delay $(t$, chaos $) \square\langle\mathrm{C}, f p\rangle$ where $\langle\mathrm{C}, f p\rangle$ is a prenormal form with each $f p(B, t)$ in superprenormal form and $\Gamma_{2}(\mathrm{C})=\emptyset$, and top $(\mathrm{C})<t$.

The last conditions reflect the special nature of chaos: when it starts executing, then everything else does not matter anymore. The next lemma states that these conditions can always be obtained:

Lemma 4.23 Let $P$ be a superextended TCSP term; in the new proof system the following formulae are true:

1. if $t \leq t^{\prime}$, then delay $(t$, chaos $) \square \operatorname{delay}\left(t^{\prime}, P\right) \equiv \operatorname{delay}(t$, chaos $)$,

2. delay $(t$, chaos $) \square \operatorname{delay} *\left(t^{\prime}, P\right) \equiv \operatorname{delay}(t$, chaos $) \square \square_{t^{\prime} \leq \ell<t} \operatorname{delay}(\ell, P)$.

Every normal form is a superprenormal form too. Superprenormal forms are determined by the instant $t$ associated to the chaotic component (delay $(t, c h a o s))$, if it exists, and by the set $\mathrm{C}$ and the function $f p$ associated to the ordinary component. We use the following notation: $\langle t, \mathrm{C}, f p\rangle$. When a superprenomal form does not have chaotic component (on the first level) we take $t=\infty$.

Notice that the previously defined concepts of expansion and distributivity do not depend on the internal structure of the $f p(B, t)$, but only on the "surface" of the prenormal form. Therefore, both concepts and its corresponding results (like any prenormal form can be expanded, etc), can be directly extended to superprenormal forms without chaotic component $(\langle\infty, \mathrm{C}, f p\rangle)$ and from these to the general ones, as any superprenormal form can be written:

$$
\langle t, \mathrm{C}, f p\rangle \equiv \operatorname{delay}(t, \text { chaos }) \square(\infty, \mathrm{C}, f p\rangle
$$

Def. $4.18 A$ term is in supernormal form if it is in distributed superprenormal form $\langle t, \mathrm{C}, f p\rangle$ with $\mathrm{C}$ convex and each $f p(B, t)$ in supernormal form.

Props. 4.24 For each superbasic term $P$ there exists a supernormal form $\langle t, \mathrm{C}, f p\rangle$ such as $\vdash_{x} P \equiv\langle t, \mathrm{C}, f p\rangle$.

Props. 4.25 Let $\langle t, \mathrm{C}, f p\rangle$ and $\left\langle t^{\prime}, \mathrm{C}^{\prime}, f p^{\prime}\right\rangle$ be supernormal forms, then

$$
\langle t, \mathrm{C}, f p\rangle \leq_{\mathrm{s}}\left\langle t^{\prime}, \mathrm{C}^{\prime}, f p^{\prime}\right\rangle \Longrightarrow \vdash_{x}\langle t, \mathrm{C}, f p\rangle \sqsubseteq\left\langle t^{\prime}, \mathrm{C}^{\prime}, f p^{\prime}\right\rangle
$$

The following theorem is a corollary of propositions 4.24 and 4.25 .

Theorem 4.26 Let $P, Q$ be superbasic terms, then $P={ }_{s} Q$ implies $\vdash_{x} P \equiv Q$.

Props. 4.27 Any superextended and finite TCSP term can be transformed into a superbasic term. 


\subsubsection{Semantic aproximations}

In order to prove the completeness with respect to recursive (guarded) processes, we will use the concept of syntactic aproximation $(\operatorname{APX}(P))$. Some way of "measuring" the degree of semantic aproximation for these syntactic aproximations is needed. To this purpose we define a second family of semantic orders, which depend on the number of instants in which visible actions are executed ${ }^{10}$. First of all we count the number of non-empty steps in a trace:

Def. 4.19 For each $t f \in \mathcal{T} \mathcal{T} \mathcal{F}$, the number of non-empty steps in tf is defined by $s t(t f)$ :

$$
\begin{aligned}
& s t\left(\left(t_{\emptyset}, 0\right)\right)=0, \\
& s t(t f+\mathrm{TB}(B, \text { end }(t f)+1))=\left\{\begin{array}{lll}
s t(t f) & \text { if } & B=B_{\emptyset} \\
s t(t f)+1 & \text { if } & B \neq B_{\emptyset}
\end{array}\right.
\end{aligned}
$$

We need observation sets with restrictions on the number of steps of the contained traces.

Def. 4.20 Let $S p \in \mathcal{S} x$ and $n \in \mathbb{N}$, we define:

$$
\begin{aligned}
F^{n}(S p)= & \left\{\langle t f, \emptyset\rangle \in F(S p) \mid s t(t f)=n \wedge \operatorname{trace}(t f)(\operatorname{end}(t f)) \neq B_{\emptyset}\right\} \\
& \cup\{\langle t f, r\rangle \in F(S p) \mid \operatorname{st}(t f)<n\} \\
D^{n}(S p)= & \left\{t f \in D(S p) \mid s t(t f) \leq n \wedge \operatorname{trace}(t f)(\operatorname{end}(t f)) \neq B_{\emptyset}\right\} \\
O B^{n}(S p)= & \left\langle F^{n}(S p), D^{n}(S p)\right\rangle .
\end{aligned}
$$

The corresponding semantic orders are defined:

Def. 4.21 Let $S p_{1}, S p_{2} \in \mathcal{S} x$ and $n \in \mathbb{N}: S p_{1} \leq^{n} S p_{2} \Leftrightarrow O B^{n}\left(S p_{2}\right) \subseteq O B^{n}\left(S p_{1}\right)$.

Lemma 4.28 Let $S p_{1}, S p_{2} \in \mathcal{S} x$, then $S p_{1} \leq_{S} S p_{2} \Leftrightarrow \forall n \in \mathbb{N}: S p_{1} \leq^{n} S p_{2}$.

We shall write $P \leq^{n} Q$ when $\mathcal{S} x \llbracket P \rrbracket \leq^{n} \mathcal{S} x \llbracket Q \rrbracket$.

Lemma 4.29 Let $P$ be a finite superextended TCSP term, and $Q$ a guarded TCSP term:

$$
P \leq \mathrm{s} Q \Longrightarrow \exists n \in \mathbb{N}: P \leq_{\mathrm{s}} Q^{n}
$$

Proof : We first prove that $\forall n \in \mathbb{N} \exists m_{n}: P \leq^{n} Q^{m_{n}}$. As $P$ is syntactically finite, there exists some instant when every computation of $P$ reaches stop or chaos. In both cases, from that instant on, $O B^{n}(P)$ is constant, and thanks to lemma 4.28 we can coniclude the proof.

Theorem 4.30 (Completeness and correctness for guarded TCSP processes) . Let $P, Q \in C R E C G\left(\Sigma_{1}\right)$, then $P \leq{ }_{\mathrm{s}} Q \Longleftrightarrow \vdash_{x} P \sqsubseteq Q$

Proof :

\footnotetext{
${ }^{10}$ The above defined orders refering to the trace length are insufficient, due to the relation between hidden actions and the delay operators.
} 
1. Correctness $(\Longleftarrow)$ is proven by proposition 4.22 .

2. In order to prove the completeness $(\Longrightarrow)$, take $P^{\prime} \in \operatorname{APX}(P)$ then $P^{\prime} \leq_{\mathrm{s}} P \leq{ }_{\mathrm{s}} Q$. By lemma 4.29 there exists $Q^{\prime} \in \operatorname{APX}(Q)$ such as $P^{\prime} \leq{ }_{\mathrm{S}} Q^{\prime}$, and by propos.4.26, we have that $\vdash_{x} P^{\prime} \sqsubseteq Q^{\prime}$. Therefore, for any $Q^{\prime} \in \operatorname{APX}(Q)$ it is true that $P^{\prime} \sqsubseteq Q^{\prime} \sqsubseteq Q$, and by rule (R2) we have that $\vdash_{x} P \sqsubseteq Q$.

\section{Related and Future Work}

We have presented a timed model suitable for real-time systems, where time is explicitely expressed by providing a global clock, so that we can see what happens at each instant and observe how the processes evolve in time.

The model induces a denotational failure semantics with divergences for TCSP processes, for which we have provided a correct and complete proof system. The view is completed by the operational approach presented in [Ort91].

Among the other existing timed models mentioned in the introduction, the most related and similar to ours is Timed TCSP [RR87], which was largely commented and compared with Timed Observations in our first work [OdF90] (a brief comparison with other timed models can be found there too and in [Ort90]). Schneider provides in [Sch90] a proof system for Timed TCSP, but his approach is very different to ours, as he is concerned with the properties of the behaviours of the processes, so that the proof system works on the basis of predicates over semantical objects instead of directly manipulating processes.

Algebraic and operational-oriented timed models like [NRSV90,QAF89,MT89,HR90, $\mathrm{BB} 90, \mathrm{Yi90}$ ] include more or less complete sets of equational laws, but as they do not consider denotational semantics, and they are more concerned with equivalences derived from bisimulations and the like, they are in general far related to our work on the proof system. Still, we would like to point out some similarities that we have found in [MT89], although this work was known to us well after our proof system was completed. Their approach is a timed extension of CCS [Mil80]. Consequently they do not distinguish between internal and external non-determinism, simplifying greatly the set of equational laws and the corresponding normal forms. Actions are instantaneous and concurrency is expressed by interleaving. The passing of time must be explicitely stated by means of a delay operator $(t) . P$, which is equivalent to our delay $(t, P)$. Although their prefix operator assumes that the prefixed action is immediately executed, they include a special delay operator $\delta . P$, which is equivalent to our $\operatorname{dela} y *(P)$, thus our prefix operator can be similarly defined as in axiom (X) (in table 4). Another difference is that their deadlock process 0 cannot witness any passage of time (unlike stop), but we have that stop is equivalent to $\delta .0$. Therefore, despite of the many differences, it is interesting to constate that most of the axioms stated there have a corresponding axiom in our proof system, and that the normal forms that they use are quite related to our normal forms (if we ignore the initial part corresponding to the internal choice).

Although the step-failure semantics presented in [TV89] is not a timed model, we found there many similarities with Timed Observations, so that it was a great help to 
refine and improve our already conceived and developed model. Moreover, the proof system presented by Taubner \& Vogler was the source of inspiration and the starting point for the present work.

As pointed out in [OdF90,Ort90], the model studied in this paper provides a too liberal treatment of time, inadequate to specify real time processes with time-outs and/or maximum parallelism requirements. Nevertheless we already introduced there (in the above cited works) two extensions of the model to cope with these features, and although more sophisticated (specially the second one) they are based on similar ideas to the original model. Therefore, several of the formal problems related to the future axiomatisation of these modified versions are already solved by the results obtained in the present work, so that the presented proof system can be taken as a basis for the development of the corresponding proof systems for the other two versions.

\section{Acknowledgements}

We thank Prof. K. Indermark (Lehrstuhl für Informatik II / RWTH-Aachen) for his support and kind hospitality. Thanks also to Paz for her help.

\section{References}

[Azc89] A. Azcorra. Modelado Formal de Sistemas Síncronos. PhD thesis, ETSI Telecomunicación, Univ.Politécnica de Madrid, 1989.

[BB90] J.C.M. Baeten and J.A. Bergstra. Real Time Process Algebra. Technical Report, CWI/ Amsterdam, 1990.

[BC88] G. Boudol and I. Castellani. Concurrency and atomicity. TCS, (59):25-84, 1988.

[BR85] S.D. Brookes and A.W. Roscoe. An improved failures model for communicating processes. In Pittsburgh Seminar on Concurrency, Springer Verlag, 1985.

[Bro83] S.D. Brookes. A Model for Communicating Sequential Processes. PhD thesis, Oxford Univ., 1983.

[GB87] R. Gerth and A. Boucher. A timed failures model for extended communicating processes. In ICALP 8\%, Springer Verlag, 1987.

[HBR81] C.A.R. Hoare, S.D. Brookes, and A.W. Roscoe. A Theory of Communicating Sequential Processes. Technical Report, Programming Research Group / Oxford Univ. (UK), 1981. Tech. Monograph PRG-16.

[Hen88] M. Hennessy. Algebraic Theory of Processes. MIT Press, 1988.

[HR90] M. Hennessy and T. Regan. A Temporal Process Algebra. Technical Report, University of Sussex (UK), 1990. 
[KSdR*85] R. Koymans, R.K. Shyamasundar, W.P. de Roever, R. Gerth, and S. ArunKumar. Compositional semantics for real-time distributed computing. In Conference on Logics of Programs, Springer Verlag, 1985.

[Mil80] R. Milner. A Calculus of Communicating Systems. LNCS 92, Springer Verlag, 1980.

[Mil83a] G.J. Milne. CIRCAL and the representation of Communication, Concurrency and Time. Technical Report, Dept.of Computer Science / Univ.Edinburgh (UK), 1983. CSR 151-83.

[Mil83b] R. Milner. Calculi for synchrony and asynchrony. TCS, (25):267-310, 1983.

[MT89] F. Moller and C. Tofts. A Temporal Calculus of Communicating Systems. Technical Report, Dept.of Computer Science / Univ.Edinburgh (UK), 1989. ECS-LFCS 89-104.

[NRSV90] X. Nicollin, J.L. Richier, J. Sifakis, and J. Voiron. ATP-an algebra for timed processes. In M. Broy and C.B. Jones, editors, TC2-Working Conference on Programming Concepts and Methods, North-Holland, 1990.

[OdF90] Y. Ortega-Mallén and D. de Frutos-Escrig. Timed observations: a semantic model for real-time concurrency. In M. Broy and C.B. Jones, editors, TC2Working Conference on Programming Concepts and Methods, North-Holland, 1990.

[OH86] E.R. Olderog and C.A.R. Hoare. Specification-oriented semantics for communicating processes. Acta Informatica, (23):9-66, 1986.

[Old86] E.R. Olderog. Process theory : semantics, specification and verification. In Advanced School on Current Trends in Concurrency, Springer Verlag, 1986.

[Ort90] Y. Ortega-Mallén. En Busca del Tiempo Perdido. PhD thesis, Fac. CC. Matemáticas, Univ.Complutense de Madrid, 1990.

[Ort91] Y. Ortega-Mallén. Timed Observations as Operational Semantics. Technical Report, Dept. Informática y Automática, Univ.Complutense Madrid (Spain), 1991. 91-1.

[QAF89] J. Quemada, A. Azcorra, and D. Frutos. A Timed Calculus for LOTOS. Technical Report, DIT, E.T.S.I. Telecomunicación / Univ. Politécnica de Madrid (Spain), 1989.

[RR87] G.M. Reed and A.W. Roscoe. Metric spaces as models for real-time concurrency. In Mathematical Foundations of Programming Language Semantics, Springer Verlag, 1987.

[Sch90] S. Schneider. Correctness and Communication in Real-Time Systems. $\mathrm{PhD}$ thesis, Oxford University Computing Laboratory (UK), 1990. 
[SM81] A. Salwicki and T. Muldner. On the algoritmic properties of concurrent programs. In Logic of Programs 1979, Springer Verlag, 1981.

[TV89] D. Taubner and W. Vogler. The step failure semantics and a complete proof system. Acta Informatica, (27):125-156, 1989.

[Yi90] Wang Yi. Real-time behaviour of asynchronous agents. In J.C.M. Baeten and J.W. Klop, editors, CONCUR'90, Theories of Concurrency: Unification and Extension, Springer Verlag, 1990. 